\title{
Computed tomography-based treatment planning for high-dose-rate brachytherapy using the tandem and ring applicator: influence of applicator choice on organ dose and inter-fraction adaptive planning
}

\author{
Vishruta A. Dumane, PhD, Yading Yuan, PhD, Ren-Dih Sheu, PhD, Vishal Gupta, MD \\ Department of Radiation Oncology, Icahn School of Medicine at Mount Sinai, New York, NY, USA
}

\begin{abstract}
Three dimensional planning for high-dose-rate (HDR) brachytherapy in cervical cancer has been highly recommended by consensus guidelines such as the American Brachytherapy Society (ABS) and the Groupe Européen de Curiethérapie - European Society for Radiotherapy and Oncology (GEC-ESTRO). In this document, we describe our experience with computed tomography (CT)-based planning using the tandem/ring applicator. We discuss the influence of applicator geometry on doses to organs at risk (OARs), namely the bladder, rectum, and sigmoid. Through example cases with dose prescribed to point $\mathrm{A}$, we demonstrate how adaptive planning can help achieve constraints to the OARs as per guidelines.
\end{abstract}

J Contemp Brachytherapy 2017; 9, 3: 279-286 DOI: https://doi.org/10.5114/jcb.2017.68519

Key words: adaptive, cervical cancer, cervix, dosimetry, HDR brachytherapy, tandem/ring.

\section{Purpose}

Intracavitary brachytherapy is critical in the management of locally advanced cervical cancer $[1,2,3,4,5,6]$. Historically, dose has been prescribed to point $A$, originally defined by the Manchester system as being $2 \mathrm{~cm}$ along the tandem starting from the level of the surface of the vaginal ovoids and then $2 \mathrm{~cm}$ perpendicular to this in the lateral direction [7]. However, due to the difficulty in visualizing the surface of the ovoids in radiographic films, Tod and Meredith modified the definition of point A with measurement along the tandem beginning at the most inferior point of the sources in the tandem, which is the flange abutting the cervical os [8]. Doses to critical organs, namely the bladder and the rectum, were documented as per the definitions by the International Commission on Radiation Units (ICRU) 38 [9]. In 2006, the Groupe Européen de Curiethérapie - European Society for Radiotherapy and Oncology (GEC-ESTRO) recommended the implementation of three dimensional (3D) magnetic resonance (MRI) image assisted brachytherapy treatment planning because of the ability to better visualize the cervix and the tumor $[10,11,12]$. A survey by the American Brachytherapy Society (ABS) in 2010 on the use of 3D imaging in gynecological brachytherapy [13], showed that the majority of physicians preferred the use of computed tomography (CT) for imaging post-implantation over the use of 2D plain radiographs for treatment planning due to improved visualization of soft tissue anatomy. Preference was also found to prescribe to point A rather than to a volume. Updated guidelines published by the ABS strongly recommended the use of 3D imaging with CT, MRI, or radiographic imaging with treatment planning performed at each fraction [14].

Guidelines published by GEC-ESTRO with MRI based planning recommend dose distribution to conform to clinical target volume (CTV) while trying to spare organs at risk (OARs). Although some institutions have routinely established the use of MRI in brachytherapy for cervical cancer, showing improvements in outcomes, with respect to normal tissue toxicity, local control, and overall survival $[15,16,17,18,19]$, initiating an MRI-guided brachytherapy treatment program for cervical cancer has its challenges $[20,21,22,23,24,25,26]$. Moreover, in some centers, lack of a dedicated MRI-simulator or accessibility of MRI from diagnostic radiology for treatment planning with the applicator in a patient, makes it necessary to use other imaging modalities such as CT. In this document, we describe adaptive CT-based planning using the tandem/ring applicator with dose prescribed to point A. Using example cases, we describe the planning process explaining the influence of applicator 
dimensions on critical organ doses, and how inter-fraction adaptive planning can help improve the ability to meet recommended dose constraints as defined by the GEC-ESTRO working group, as well as in more recent trials and task groups, specifically European Study on MRI - Guided Brachytherapy in Locally Advanced Cervical Cancer (EMBRACE) [27], ABS [14], and Cancer Care Ontario (CCO) [28].

\section{Procedure}

Patients were treated with external beam radiotherapy (EBRT) to 45 Gy or 50.4 Gy in 1.8 Gy fractions via three-dimensional conformal radiation therapy (3D CRT) with concurrent cisplatin and HDR brachytherapy interdigitated to complete the treatment typically within 8 weeks. A parametrial boost was occasionally administered to an additional 5.4-9 Gy in 3-5 fractions if needed. Fractionation regimens for HDR varied among our patients: 30 Gy in 5 fractions, 27.5 Gy in 5 fractions, 28 Gy in 4 fractions, or 24 Gy in 3 fractions. The exact fractionation schedule was based on tumor size, tumor response to EBRT and chemotherapy, and patient compliance. At our institution, at the first fraction, a Smitt sleeve is sutured at the external os under anesthesia in the operating room. The Smitt sleeve facilitates tandem insertion and acts as a marker for the cervical os as shown in Figure 1. For tandem and ring applicators from Elekta (Elekta company, Elekta AB, Stockholm, Sweden), the available applicator angles are $30^{\circ}, 45^{\circ}$, and $60^{\circ}$; available ring diameters are $26 \mathrm{~mm}, 30 \mathrm{~mm}$, and $34 \mathrm{~mm}$, and accessible tandem lengths are $20 \mathrm{~mm}, 40 \mathrm{~mm}$, and $60 \mathrm{~mm}$. The choice of the applicator angle, ring diameter, and tandem length for the first fraction are decided based on patient anatomy. The uterus length is measured just prior to the Smitt sleeve insertion and this measurement determines the tandem length. The largest ring that can fit is typically used. The angle is determined based on the cervix/uterus position in the pelvis. Ideally, the tandem bisects the uterus on sagittal view. The applicator, along with the rectal retractor, is inserted in the patient followed by placement of vaginal packing anteriorly to push the bladder away from the applicator. After the patient recovers from the procedure, a CT scan is acquired at $3 \mathrm{~mm}$ slice spacing and images transferred to the treatment planning system. Applicator insertions for the following fractions are performed as outpatient procedures. CT images are acquired, and a new treatment plan is performed for each insertion/fraction.

\section{Treatment planning}

The software used for treatment planning in this document is Oncentra version 3.3 SP 3 (Elekta, Stockholm, Sweden). After CT images are acquired and transferred to the planning system, the bladder, rectum, and sigmoid are contoured followed by reconstruction of the applicator. The origin of the applicator is placed at the Smitt sleeve as in Figure 2A to mark the cervical os with axes aligned as shown. The tandem and ring are reconstructed starting from the tip end of each applicator followed by activation of the dwell positions. The dwell positions in the ring are activated on the sides at the vaginal fornices to mimic the ovoids, as shown in Figure 2B. The tandem is activated from the first dwell position at the tip extending inferiorly to stop above the plane of the ring, making sure not to extend past the origin or the cervical os. The dose is then prescribed to point $\mathrm{A}$ in the coordinate system of the applicator $2 \mathrm{~cm}$ from the origin, along the tandem, and $2 \mathrm{~cm}$ perpendicular to it on either side. The plan is normalized such that $100 \%$ of the prescription dose is delivered to point A. Optimization is achieved manually by
A

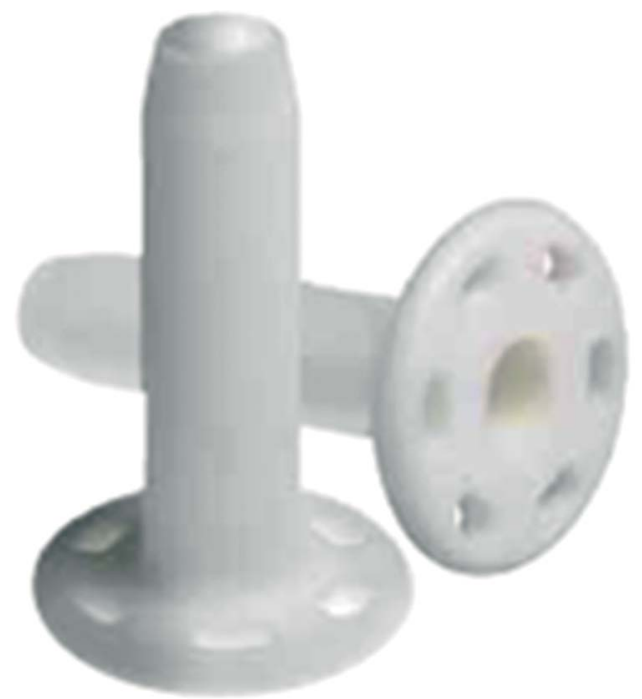

\section{B}

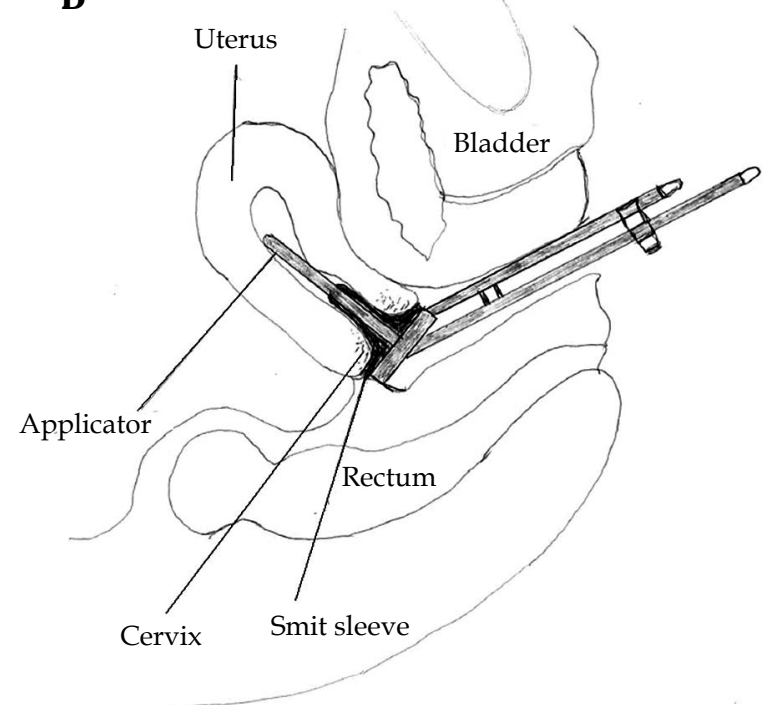

Fig. 1. A) The Smitt sleeve is a plastic tube with drainage holes that is inserted into the cervical OS and sutured in place prior to the first treatment. It helps to keep the cervix dilated for subsequent applicator insertions throughout the course of treatment. B) Illustration of the Smitt sleeve and the applicator inside a patient. The Smitt sleeve helps guide the insertion of the tandem and also acts as a stopper for the ring at the cervix 

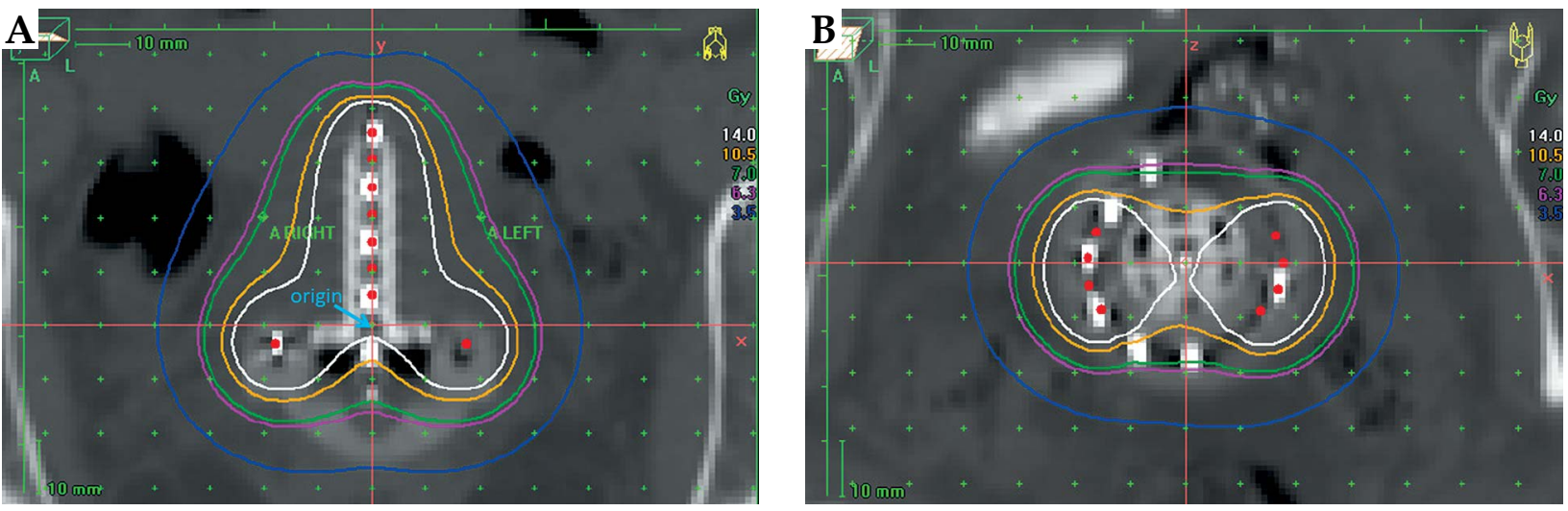

Fig. 2. A) The origin of the applicator (as indicated by the arrow) is placed at the Smitt sleeve to mark the cervical os with axes aligned as shown. The tandem is activated from the first dwell position at the tip extending inferiorly to stop above the plane of the ring making sure not to extend past the origin or the cervical os. Dose is prescribed to point $\mathrm{A}$ in the co-ordinate system of the applicator $2 \mathrm{~cm}$ from the origin along the tandem and $2 \mathrm{~cm}$ perpendicular to it on either side. The plan is normalized such that $100 \%$ of the prescription dose goes to point A. The brachytherapy dose prescribed in this case was 7 Gy $x 4$ fractions. B) The dwell positions in the ring are activated typically 4 to 5 on the sides at the vaginal fornices to mimic the ovoids as shown

adjusting the activated dwell positions and their weights. Manual optimization suffices to create a 'pear shaped' dose distribution. Usually, the dwell weight at the tip of the tandem is greater than the rest of the tandem to help increase coverage laterally [29]. As a starting point, at our institution the weight on the first dwell position at the tip of the tandem is 1.0, the intermediate dwell positions in the tandem are weighed 0.75 , and the dwell position closest to the ring is weighed 0.5 . The weight on all the dwell positions in the ring is 0.5 . The cumulative dose volume histogram (DVH) is computed for the bladder, rectum, and sigmoid, and the weights adjusted to optimally minimize dose to these critical organs.

\section{Dose constraints and recommendations}

The biological effect of the administered radiotherapy dose depends not only on the dose distribution, but also on the dose rate, fractionation, and duration of the treatment. In order to take into account the differences in time dose fractionation patterns of both pelvic external beam radiotherapy and intracavitary brachytherapy, the biologically effective dose (BED) using the linear quadratic (LQ) model is calculated separately for both cases using the equation given by:

$$
B E D=N d\left(1+g \frac{d}{\alpha / \beta}\right)
$$

where $N$ is the number of fractions, $d$ is the dose per fraction, $\alpha / \beta=3$ for normal tissues, and $g$ is a function that accounts for incomplete repair. For external beam as well as HDR brachytherapy, $g=1$ [12]. From the BED, the equivalent external beam therapy dose using conventional fractionation of $2 \mathrm{~Gy}$ per day $\left(\mathrm{EQD}_{2}\right)$ is computed as:

$$
E Q D_{2}=\frac{B E D}{1+\frac{2}{\alpha / \beta}}
$$

For each brachytherapy fraction, the dose to the hottest $2 \mathrm{cc}\left(\mathrm{D}_{2 \mathrm{cc}}\right)$ is obtained for the bladder, rectum, and sigmoid from the DVH. The BED and $\mathrm{EQD}_{2}$ are calculated for each of OARs as per equations 1 and 2 for each fraction, and the cumulative $\mathrm{EQD}_{2}$ value of the $\mathrm{D}_{2 \mathrm{cc}}$ for each OAR combining external beam therapy and brachytherapy over all fractions is obtained. The constraints recommended for the cumulative $D_{2 c c}$ by GEC-ESTRO, EMBRACE, ABS, and CCO are < 90 Gy $\mathrm{EQD}_{2}$ for the bladder, and $<70-75$ Gy $\mathrm{EQD}_{2}$ for the rectum and sigmoid. This formalism is of course dependent on the assumption that full dose of external beam therapy is administered in the volume of interest, identical location during fractionated brachytherapy, and contiguous contouring of volumes for all the OARs. If $C$ denotes the $\mathrm{EQD}_{2}$ constraint for the combined external beam and brachytherapy plans for a given OAR, then the allowable $\mathrm{D}_{2 \mathrm{cc}}$ per fraction of brachytherapy $\left(d_{B}\right)$ for that OAR can be calculated as follows:

$$
C=E Q D_{2 E}+E Q D_{2 B}
$$

where $E Q D_{2 E}$ is contribution from external beam and $E Q D_{2 B}$ is from all fractions of brachytherapy

$$
\begin{gathered}
C=\frac{B E D_{E}+B E D_{B}}{1+\frac{2}{\alpha / \beta}} \\
C=\frac{B E D_{E}+N_{B} d_{B}\left(1+g \frac{d_{B}}{\alpha / \beta}\right)}{1+\frac{2}{\alpha / \beta}}
\end{gathered}
$$

From equation (1), $B E D_{E}$ is 72 Gy for a dose of $45 \mathrm{~Gy}$ in $1.8 \mathrm{~Gy}$ fractions, and 80.6 for $50.4 \mathrm{~Gy}$ in $1.8 \mathrm{~Gy}$ fractions. Substituting the values for $C, B E D_{E}, N_{B}, g=1$, and $\alpha / \beta=3$, the value of $d_{B}$ can be obtained and serves as a dose constraint for the respective OAR for that fraction. Depending on the ability to meet this constraint in the first fraction, the value of $d_{B}$ for the next fraction can vary such that the cumulative $\mathrm{EQD}_{2}$ can be maintained within the value of the constraint $C$. Between fractions, variations in normal tissue doses are expected. Treatment planning 
by prescribing dose to point A can make it challenging to remain within the recommended tolerance doses for critical organs, and careful considerations with respect to choice of applicator dimensions, plan optimization, and dose prescription need to be made.

\section{Adaptive planning strategy}

In our preliminary retrospective analysis on more than 50 plans from 13 patients, significant correlation $(p<0.05)$ was seen between the angle of the applicator and $\mathrm{D}_{2 \mathrm{cc}}$ of the bladder, rectum, and sigmoid [30]. The higher the angle, the more posterior the applicator, thereby, increasing the rectal and the sigmoid dose, while decreasing dose to the bladder as illustrated in Figure 3. Significant correlation was also noted between $\mathrm{D}_{2 \mathrm{cc}}$ of the rectum and sigmoid, and the diameter of the ring. Larger ring diameters help push the rectum and sigmoid away from the applicator origin; thus, decreasing dose to these
A

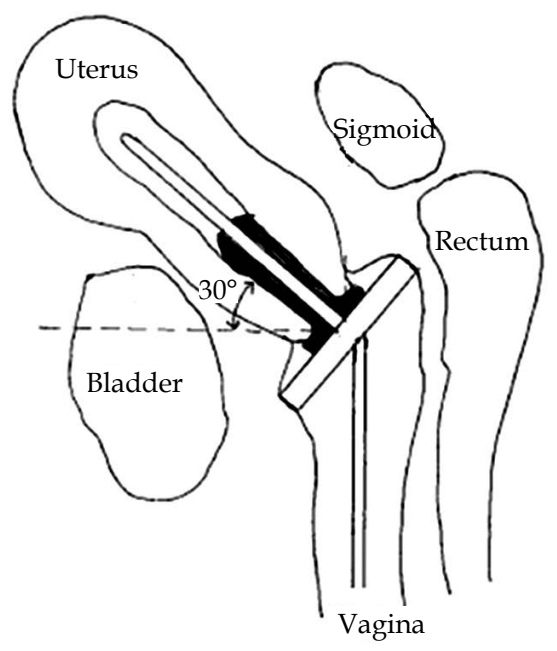

B

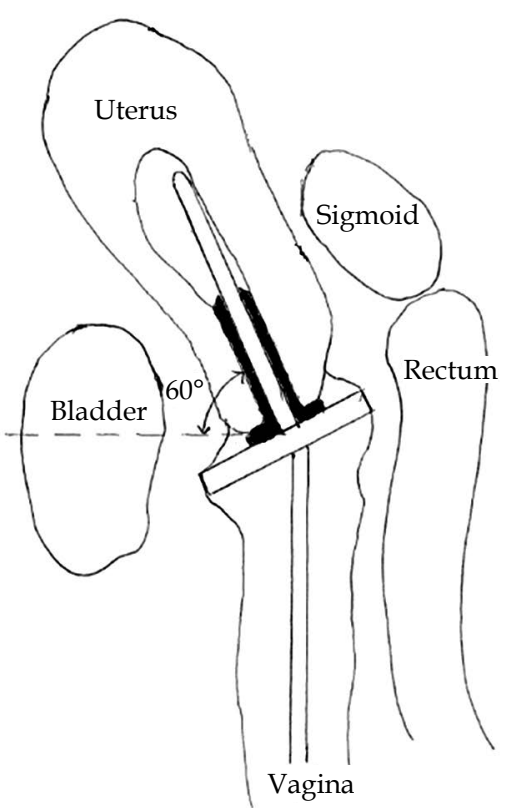

Fig. 3. A) Illustration of how the choice of the appropriate applicator angle helps spare the bladder, rectum, and sigmoid. A $30^{\circ}$ applicator is more anterior, thus potentially increasing dose to the bladder. B) A $60^{\circ}$ applicator is more posterior, thus potentially increasing dose to the rectum and sigmoid

A

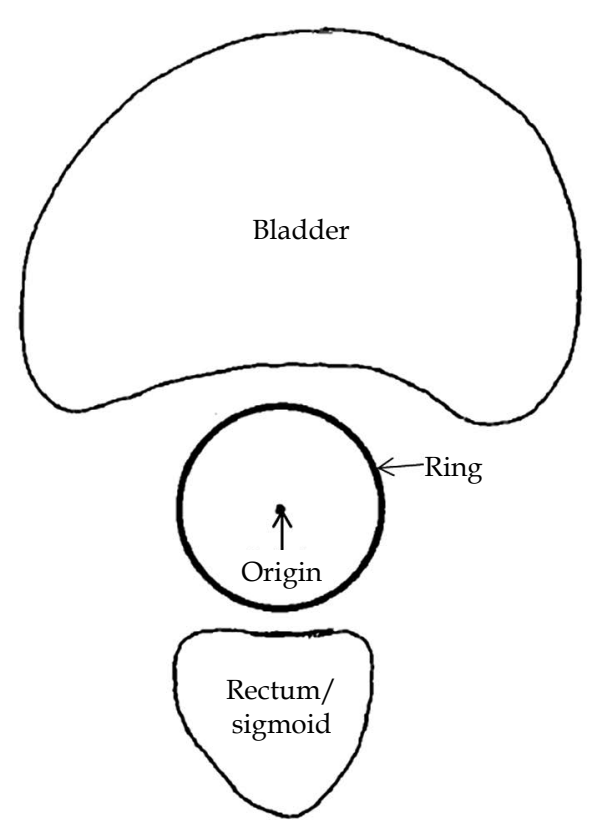

B

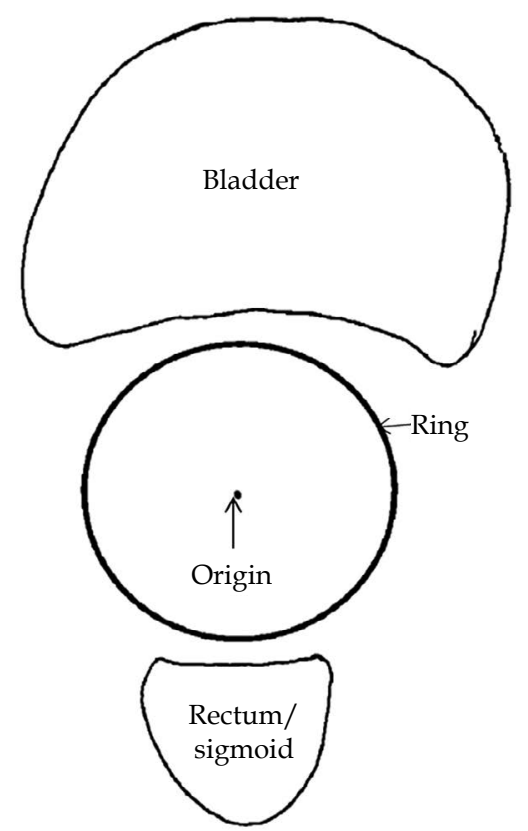

Fig. 4. A) Illustration of how the choice of the ring diameter influences dose to the rectum, sigmoid, and bladder. When a smaller ring is used, all the organs tend to be closer to the applicator origin. B) With the use of a larger ring, the rectum, sigmoid, and bladder tend to be pushed away further from the applicator origin, thereby helping to reduce their dose 
organs as illustrated in Figure 4. Therefore, choosing the applicator angle and ring diameter appropriately for the subsequent fractions can help reducing OAR doses, and accordingly helping to meet the dose constraints. During applicator insertion for the first fraction, the physician tries to maximize the choice of the ring diameter, while using the rectal retractor and anterior vaginal packing. The choice of the applicator angle depends on the uterus position within the pelvis (anteverted, neutral, or retroverted). Optimization is achieved manually by adjusting the dwell positions in the ring, tandem, and the dwell weights. From the DVH, the $\mathrm{D}_{2 \mathrm{cc}}$ is noted for each $\mathrm{OAR}$, and if it is less than the value of $d_{B}$ (which is the maximum allowable $\mathrm{D}_{2 \mathrm{cc}}$ for the respective OAR in the given fraction), then we can continue with the same applicator
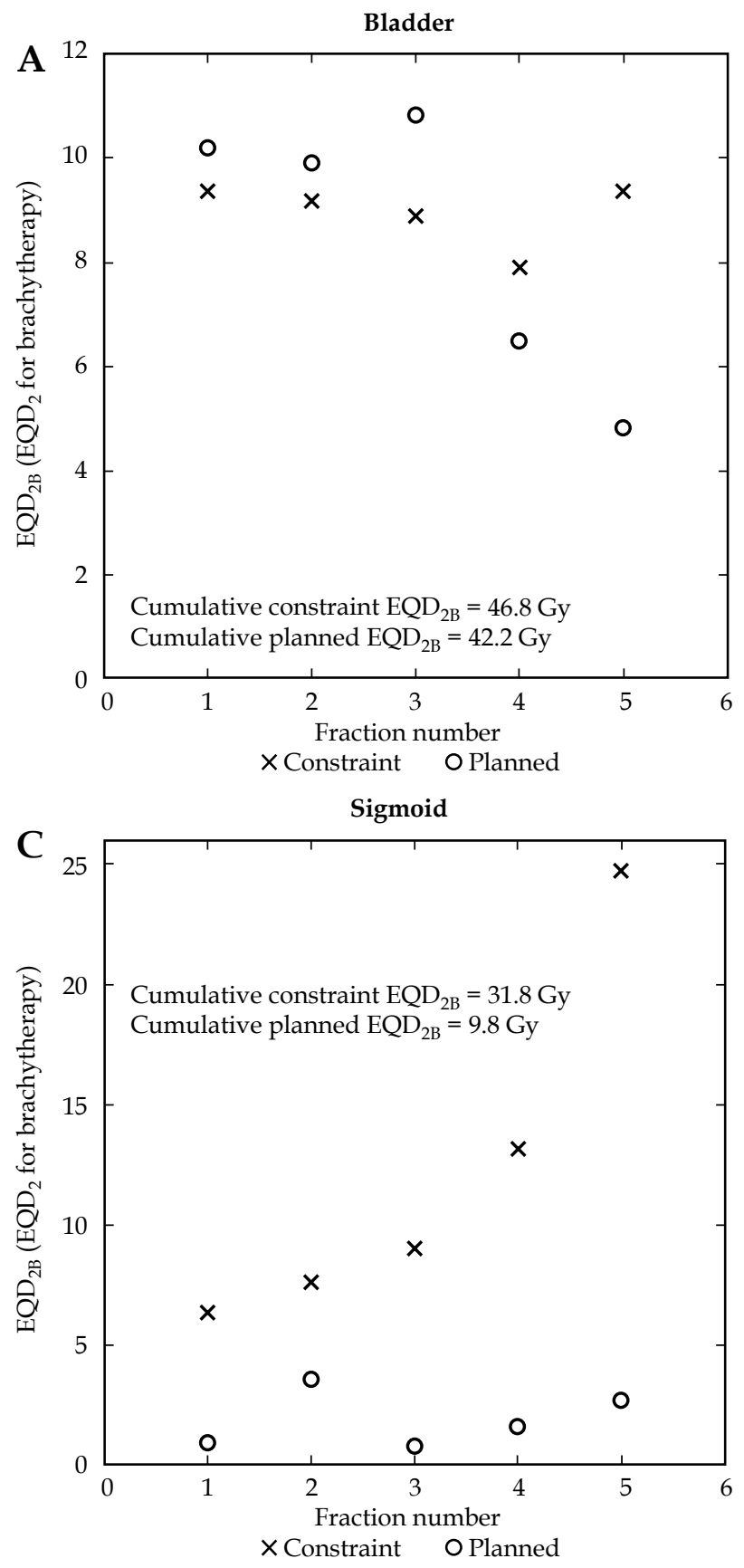

for the next fraction. However, if one or more OARs have exceeded their respective $d_{B}$ values, then appropriate adjustment of the applicator dimension will be required for the following fraction, so as to meet the cumulative $\mathrm{EQD}_{2}$ constraint $C$ for each OAR. We describe 2 example cases to explain the adaptive planning process. Depending on the institution's policies, the allowable dose $d_{B}$ may be calculated using an in-house software program, although excel spreadsheets from the Department of Radiotherapy and Radiobiology, Medical University of Vienna can be downloaded at: https://www.americanbrachytherapy. org/guidelines/LQ_spreadsheet.xls.

The choice of the applicator dimensions for each treatment fraction, however, is at the discretion of the treating physician.

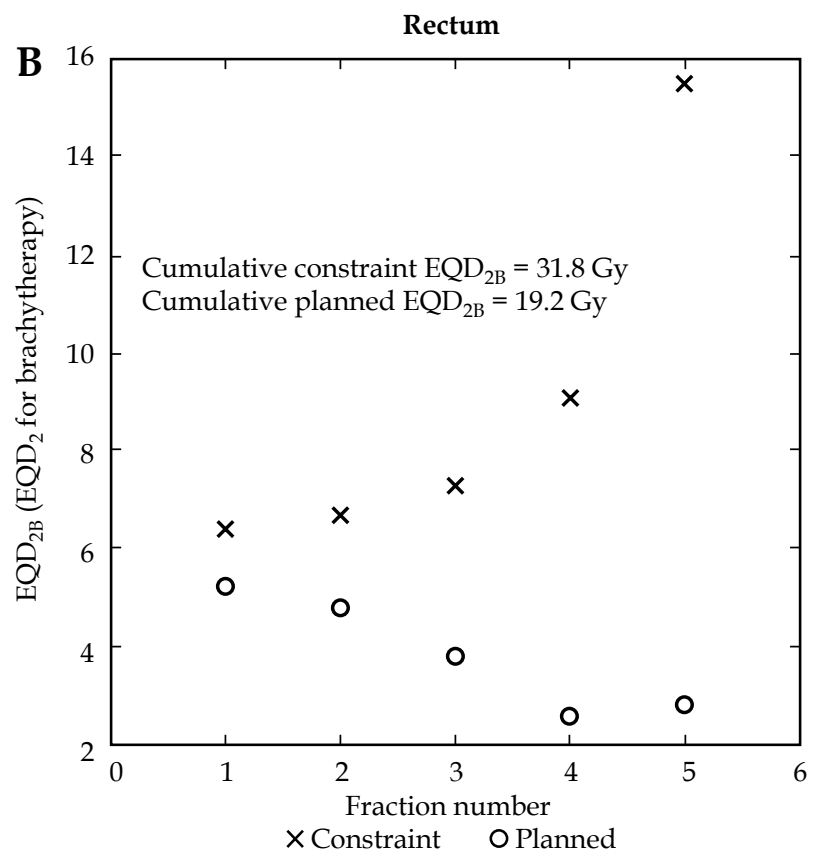

Fig. 5. Adaptive planning for case 1. A) In the first 3 fractions, the applicator angle used was $30^{\circ}$, and the bladder dose in the plan was larger than the $\mathrm{EQD}_{2}$ constraint in the respective fraction. Using a $45^{\circ}$ applicator in the $4^{\text {th }}$ and $5^{\text {th }}$ fractions helped reduce the bladder dose, thereby meeting the constraint in those fractions as well as cumulatively. B) In the first 3 fractions, the rectal dose was within tolerance. Using a $45^{\circ}$ applicator in the $4^{\text {th }}$ and $5^{\text {th }}$ fractions did not increase the rectal dose for this case. C) In the first 3 fractions, the sigmoid dose was within tolerance. Using a $45^{\circ}$ applicator in the $4^{\text {th }}$ and $5^{\text {th }}$ fractions did not increase the sigmoid dose for this case 


\section{Example case 1: meeting the bladder constraint by adjusting the applicator angle}

Case 1 was prescribed 45 Gy with EBRT to the pelvis via 3CDRT and 5.5 Gy $\times 5$ fractions with tandem/ring brachytherapy. For the first fraction, the applicator angle chosen was $30^{\circ}$ with a tandem of length $4 \mathrm{~cm}$ and a ring diameter of $3 \mathrm{~cm}$. The allowable $\mathrm{EQD}_{2}$ in the first fraction was $9.4 \mathrm{~Gy}$ for the bladder and $6.4 \mathrm{~Gy}$ for the rectum as well as the sigmoid. The $\mathrm{EQD}_{2}$ for the bladder exceeded this limit in the first fraction at 10.2 Gy. However, both the rectum and sigmoid were within tolerance at $5.2 \mathrm{~Gy}$ and $1 \mathrm{~Gy}$, respectively. Results for the adaptive plan are shown in Figure 5. In the second as well as in the third fraction, the $\mathrm{EQD}_{2}$ for the bladder exceeded the limiting value, but was within tolerance for the rectum as well as

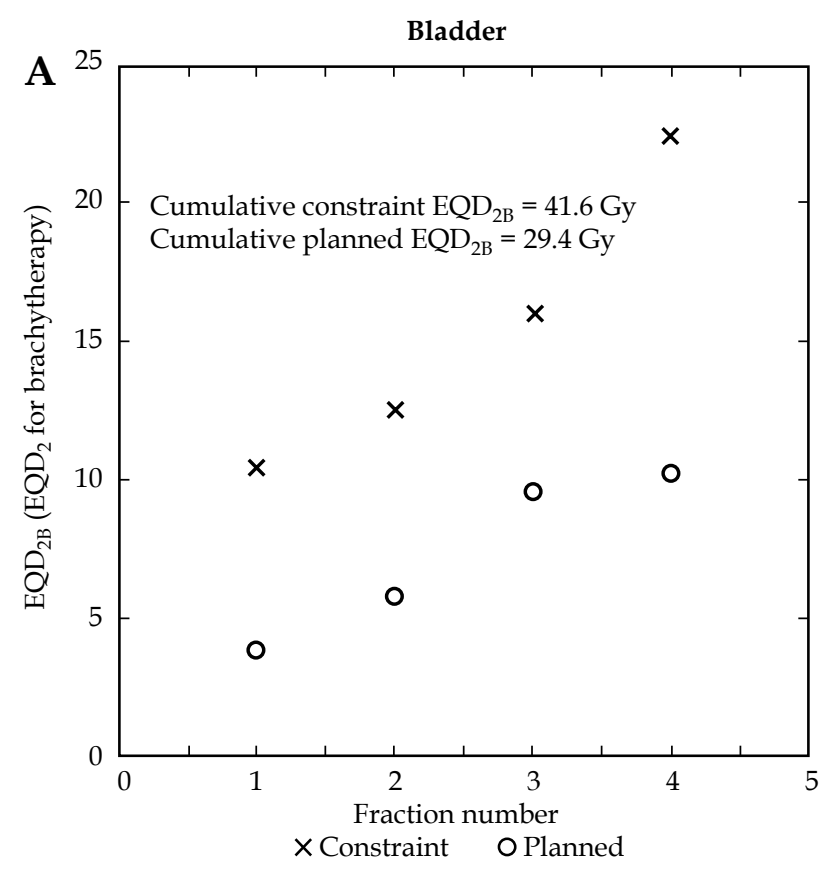

Sigmoid

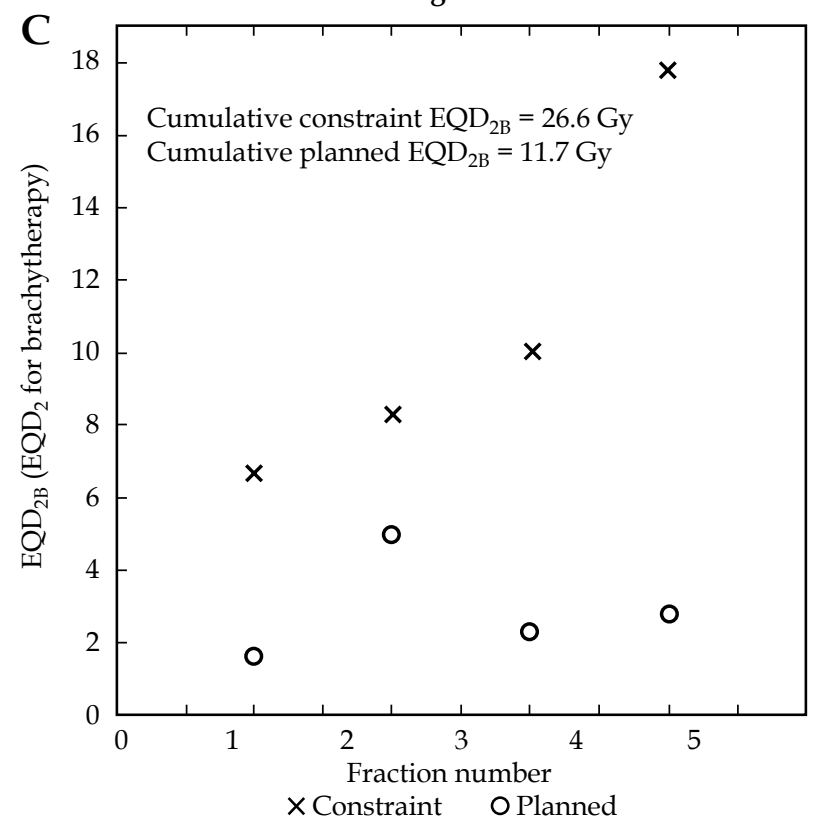

the sigmoid. For the $4^{\text {th }}$ and $5^{\text {th }}$ fractions, the angle of the applicator was changed to $45^{\circ}$, and in each of these two fractions, the $\mathrm{EQD}_{2}$ for the bladder was reduce to below the respective constraint in each. It is worth pointing out that since the constraint was always met for the rectum and the sigmoid, the limiting value of $\mathrm{EQD}_{2}$ was higher than the value in the previous fraction. The cumulative $\mathrm{EQD}_{2}$ combining EBRT and brachytherapy for the bladder in this case was $85.4 \mathrm{~Gy}<90 \mathrm{~Gy}$, and for the rectum and sigmoid at $62.2 \mathrm{~Gy}<75$ Gy and $53 \mathrm{~Gy}<75$ Gy, respectively, thereby meeting all the OARs constraints recommended $[14,15,16,19,27,28]$. The increase in applicator angle in this case, however, was not found to increase $\mathrm{D}_{2 \mathrm{cc}}$ to the rectum and sigmoid. Our analysis on average has shown that the decrease in bladder $D_{2 c c}$ when the applicator angle is changed from $30^{\circ}$ to $45^{\circ}$, is more than

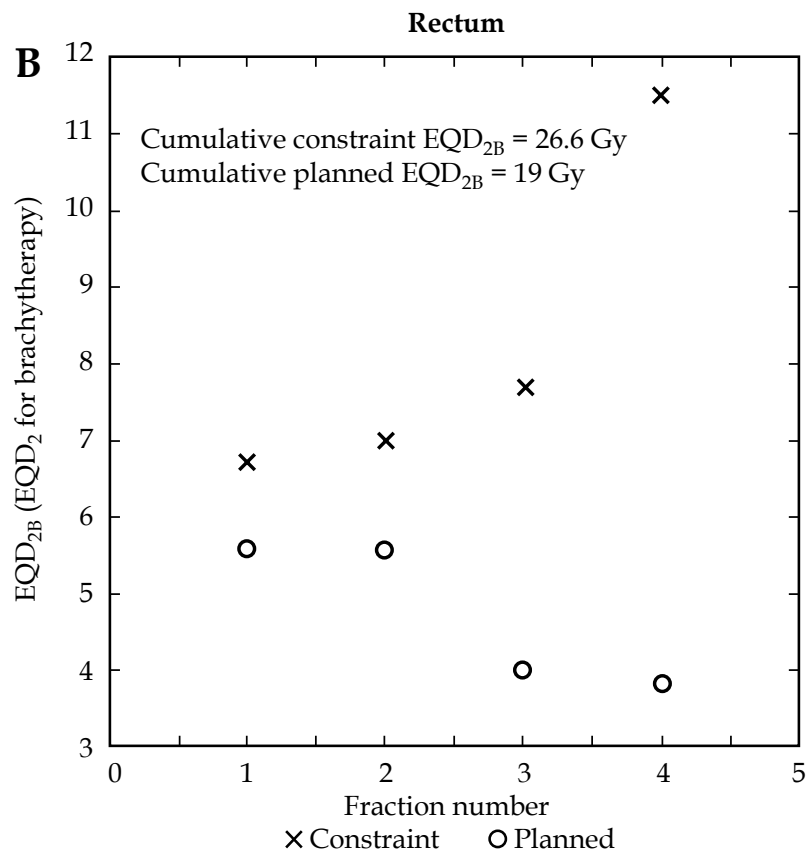

Fig. 6. Adaptive planning for case 2. A) In the first fraction, the applicator angle used was $60^{\circ}$, and $45^{\circ}$ in the second fraction. The ring diameter in both these fractions was $3 \mathrm{~cm}$ and tandem length of $4 \mathrm{~cm}$. As expected, the dose to the bladder increased. However, since the bladder constraint was met in the first fraction, the allowable limit in the next fraction was greater than in the previous. B) Reducing the applicator angle in this case did not help decrease the rectal dose. Increasing the ring diameter to $3.4 \mathrm{~cm}$ in the third fraction saw an improvement in rectal sparing. C) The sigmoid has displayed a similar trend as the rectum 
the increase in $\mathrm{D}_{2 \mathrm{cc}}$ to the rectum and sigmoid. When the applicator angle is changed from $45^{\circ}$ to $60^{\circ}$, the bladder $\mathrm{D}_{2 \mathrm{cc}}$ decreases less compared to the increase in $\mathrm{D}_{2 \mathrm{cc}}$ to the rectum and sigmoid.

\section{Example case 2: reducing the rectal dose by adjusting the ring diameter}

Case 2 was prescribed 50.4 Gy with EBRT to the pelvis via 3DCRT and 7 Gy $\times 4$ fractions with brachytherapy. The applicator chosen for the first fraction was $60^{\circ}$ with a tandem of length $4 \mathrm{~cm}$ and a ring diameter of $3 \mathrm{~cm}$. The allowable $\mathrm{EQD}_{2}$ in the first fraction was $10.4 \mathrm{~Gy}$ for the bladder and $6.7 \mathrm{~Gy}$ for both the rectum and the sigmoid. As shown in Figure 6, doses to all the OARs were below their respective limits in the first fraction. However, the rectal constraint was relatively close to the allowable limit. It was felt that this could be reduced in the following fraction and therefore, the choice of the applicator was changed from $60^{\circ}$ to $45^{\circ}$. Although this was expected to increase dose to the bladder, since the $\mathrm{EQD}_{2}$ for the bladder was well within tolerance in the first fraction, violation of this constraint while using a more anterior applicator was less of a concern for the bladder. As seen in Figure $6 \mathrm{~B}$, the $45^{\circ}$ applicator did not reduce the dose to the rectum, although it was still within the tolerance for $\mathrm{EQD}_{2}$ for that fraction. The bladder dose increased as expected, but was also within tolerance for the second fraction. For the following two fractions, the applicator angle was kept at $45^{\circ}$, but with an increased ring diameter to $3.4 \mathrm{~cm}$ (the largest available in the kit). A larger ring was used for the last 2 fractions, since the cervical tumor had shrunk and the vagina was able to accommodate a larger ring. This helped reduce the rectal dose for the following two fractions compared with the previous two cases. The sigmoid dose in the case was not concerning and was met well within tolerance for each fraction. The cumulative $\mathrm{EQD}_{2}$ for the rectum was $67.3 \mathrm{~Gy}<75 \mathrm{~Gy}$, and for the bladder and sigmoid at 77.8 Gy $<90$ Gy and 60 Gy < 75 Gy, respectively.

\section{Conclusions}

We have described treatment planning using the tandem/ring applicator with CT imaging and dose prescribed to point A. Although it is recommended to use MRI for outlining and planning, not every institution may have access or the logistical capability to use MRI for planning. With CT, organs at risk, namely the bladder, rectum, and sigmoid are well visualized as are the applicators. The definition of high-risk clinical target volume can be challenging. However, studies have shown that the greatest discrepancy between CT versus MR contours were when there was parametrial extension, but with good response from treatment prior to the use of brachytherapy. When there was no parametrial extension or there was poor parametrial response, the CT and MR contours were either identical or similar [31]. Through adaptive planning, we have explained how the applicator angle and ring diameter can be modified between fractions to reduce doses to critical organs while meeting the constraints. Dose to the bladder and to the rectum is expected to decrease and increase with the applicator angle, respectively. Increased ring diameter is expected to improve rectum and sigmoid sparing. Although larger diameters also help push the bladder more anterior and away from the origin, in our study we did not see a correlation between the $\mathrm{D}_{2 \mathrm{cc}}$ of the bladder and the ring diameter. This could be attributed to the fact that with injection of saline and contrast for imaging purposes, the bladder tends to sag laterally making it come close to the ring. Moreover, the dwell positions in the ring are activated laterally. Hence, even though larger ring diameters push the bladder further away from the origin, this advantage tends to be offset by parts of the bladder coming close to the ring laterally by the activated dwell positions. We have described how careful considerations through applicator choice in adaptive planning can help achieve dose constraints recommended by GEC-ESTRO. However, this also depends on the change of anatomy from fraction to fraction, and a combination may have to be tried to see if it helps reduce dose to the concerned OAR.

\section{Disclosure}

Authors reports no conflict of interest.

\section{References}

1. Lanciano RM, Won M, Coia LR et al. Pretreatment and treatment factors associated with improved outcome in squamous cell carcinoma of the uterine cervix: a final report of the 1973 and 1978 patterns of care studies. Int J Radiat Oncol Biol Phys 1991; 20: 667-676.

2. Eifel PJ, Morris M, Oswald MJ. The influence of tumor size and growth habit on outcome of patients with FIGO stage $1 \mathrm{~B}$ squamous cell carcinoma of the uterine cervix. Int J Radiat Oncol Biol Phys 1993; 27: 127-128.

3. Montana GS, Fowler WC, Varra MA et al. Carcinoma of the cervix, stage III: Results of radiation therapy. Cancer 1986; 57: 148-154.

4. Patel FD, Sharma SC, Negi PS et al. Low dose rate vs. high dose rate brachytherapy in the treatment of carcinoma of the uterine cervix: a clinical trial. Int J Radiat Oncol Biol Phys 1994; 28: 335-341.

5. Hareyama M, Sakata K, Oouchi A et al. High-dose-rate versus low-dose-rate intracavitary therapy for carcinoma of the uterine cervix: a randomized trial. Cancer 2002; 94: 117-124.

6. Lertsanguansinchai P, Lertbutsayanukul C, Shotelersuk K et al. Phase III randomized trial comparing LDR and HDR brachytherapy in treatment of cervical carcinoma. Int J Radiat Oncol Biol Phys 2004; 59: 1424-1431.

7. Tod M, Meredith WJ. A dosage system for use in the treatment of cancer of the uterine cervix. Br J Radiol 1938; 11: 809-824.

8. Tod M, Meredith WJ. Treatment of cancer of the cervix uteri a revised Manchester method. Br J Radiol 1953; 26: 252-257.

9. ICRU report 38. Dose and volume specification for reporting intracavitary therapy in gynecology. International Commission on Radiation Units and Measurements, Bethesda 1985; 1-20.

10. Haie-Meder C, Pötter R, Van Limbergen E et al. Recommendations from Gynaecological (GYN) GEC-ESTRO Working Group (I): Concepts and terms in 3D image based 3D treatment planning in cervix cancer brachytherapy with emphasis on MRI assessment of GTV and CTV. Radiother Oncol 2005; 74: 235-245. 
11. Lang S, Nulens A, Briot E et al. Intercomparison of treatment concepts for MR image assisted brachytherapy of cervical carcinoma based on GYN GEC-ESTRO recommendations. Radiother Oncol 2006; 78: 185-193.

12. Pötter R, Haie-Meder C, Van Limbergen E et al. Recommendations from gynaecological (GYN) GEC ESTRO working group (II): Concepts and terms in 3D image-based treatment planning in cervix cancer brachytherapy-3D dose volume parameters and aspects of 3D image-based anatomy, radiation physics, radiobiology. Radiother Oncol 2006; 78: 67-77.

13. Viswanathan AN, Erickson BA. Three-dimensional imaging in gynecologic brachytherapy: A survey of the American Brachytherapy Society. Int J Radiat Oncol Biol Phys 2010; 76: 104-109.

14. Viswanathan AN, Beriwal S, De Los Santos JF et al. The American Brachytherapy Society Treatment Recommendations for Locally Advanced Carcinoma of the Cervix Part II: High Dose-Rate Brachytherapy. Brachytherapy 2012; 11: 47-52.

15. Lindegaard JC, Fokdal LU, Nielsen SK et al. MRI-guided adaptive radiotherapy in locally advanced cervical cancer from a Nordic perspective. Acta Oncol 2013; 52: 1510-1519.

16. Pötter R, Georg P, Dimopoulos JC et al. Clinical outcome of protocol based image (MRI) guided adaptive brachytherapy combined with 3D conformal radiotherapy with or without chemotherapy in patients with locally advanced cervical cancer. Radiother Oncol 2011; 100: 116-123.

17. Pötter R, Dimopoulos J, Georg P et al. Clinical impact of MRI assisted dose volume histogram adaptation and dose escalation in brachytherapy of locally advanced cervix cancer. Radiother Oncol 2007; 83: 148-155.

18. Wang $X, \mathrm{Li} J$, Wang $P$ et al. Image guided radiation therapy boost in combination with high-dose-rate intracavitary brachytherapy for the treatment of cervical cancer. J Contemp Brachytherapy 2016; 8: 122-127.

19. Carvalho H, Mendez L, Stuart S et al. Implementation of image-guided brachytherapy (IGBT) for patients with uterine cervix cancer: a tumor volume kinetics approach. J Contemp Brachytherapy 2016; 8: 301-307.

20. Owrangi AM, Prisciandaro JI, Soliman A et al. Magnetic resonance imaging-guided brachytherapy for cervical cancer: initiating a program. J Contemp Brachytherapy 2015; 7: 417-422.

21. Yanasak N, Clarke G, Stafford RJ et al. Parallel imaging in MRI: Technology, Applications and Quality Control. 2015. American Association of Physicists in Medicine (AAPM), Task Group 118.

22. Stanescu T, Wachowicz K, Jaffray DA. Characterization of tissue magnetic susceptibility-induced distortions for MRIgRT. Med Phys 2012; 39: 7185-7193.

23. Tanderup K, Nesvacil N, Pötter R et al. Uncertainties in image guided adaptive cervix cancer brachytherapy: impact on planning and prescription. Radiother Oncol 2013; 107: 1-5.

24. Petrič P, Hudej R, Rogelj P et al. Uncertainties of target volume delineation in MRI guided adaptive brachytherapy of cervix cancer: a multi-institutional study. Radiother Oncol 2013; 107: 6-12.

25. Hellebust TP, Tanderup K, Lervag C et al. Dosimetric impact of interobserver variability in MRI-based delineation for cervical cancer brachytherapy. Radiother Oncol 2013; 107: 13-19.

26. Nesvacil N, Tanderup K, Hellebust TP et al. A multicentre comparison of the dosimetric impact of inter- and intra-fractional anatomical variations in fractionated cervix cancer brachytherapy. Radiother Oncol 2013; 107: 20-25.

27. EMBRACE Study Committee. EMBRACE: A European Study on MRI-guided brachytherapy in locally advanced cervical cancer. Available at: https://www.embracestudy. $\mathrm{dk} /$ About.aspx.

28. Cancer Care Ontario; Imaging Strategies for Definitive Intracavitary Brachytherapy of Cervical Cancer. 2014; Available at:
https://www.cancercare.on.ca/common/pages/UserFile. aspx?fileId $=309545$.

29. American Association of Physicists in Medicine Medical Physics Monograph. Accessed: 31 November 2005.

30. Dumane V, Rhome R, Yuan $Y$ et al. Influence of Applicator Dimensions on Doses to Bladder, Rectum and Sigmoid in HDR Brachytherapy for Cervical Cancer. Med Phys 2015; 42: 3447.

31. Viswanathan AN, Erickson B, Gaffney DK et al. Comparison and consensus guidelines for delineation of clinical target volume for CT- and MR-based brachytherapy in locally advanced cervical cancer. Int J Radiat Oncol Biol Phys 2014; 90: 320-328. 